\title{
Optimization of Pattern Recognition of Hijaiyah Letters using Normalized Cross Correlation Tchniques
}

\author{
Sufiatul Maryana, Iyan Mulyana, Ema Kurnia
}

\begin{abstract}
The development of analysis in digital image increasingly developed with various methods, one of which is in recognition of letter patterns. Each letter written using handwriting must have different writing patterns, such as the thickness and shape of the letter pattern. This research will be doing on the pattern recognition of hijaiyah letters of handwriting by applying the Normalized Cross Correlation (NCC) technique. NCC is a technique used to match two images. Before the NCC process, it should be done with the preprocessing using convolution and without convolution using the binary image. The convolution technique used was the Sobel and Prewitt edge detection with the aimed to get the edge of an object and compared the number of matching letters between using edge detection and without edge detection. The tests were done by using the different sized image of $32 \times 32$ pixels, $64 x 64$ pixels and then match it against a similar sample data, a different sample data, a different objects font sample data and a different sample data of original image size. The results show that the matching of the letter pattern depends on the size of the image that is more matches to the image of 32x32 pixels. The binary image had better matching numbers than the convolution techniques. While in convolution techniques, Prewitt edge detection had the higher accuracy and matching results compared to the image using Sobel edge detection.
\end{abstract}

Index Terms: Normalized Cross, Correlation Pattern Recognition, Hijaiyah Letters, Convolution, Edge Detection.

\section{INTRODUCTION}

The development of analysis in digital image increasingly developed with various methods, one of which is in the analysis and objects recognition. Computer devices can recognize a data into information, one of which is image data. Images canbe processed by computer and generate information by using certain methods called as image processing. Utilization of computer in this image processing was the object of digital image. Digital images are images that can be displayed on a computer screen and can be obtained from the scan, camera photos, satellite images, CT scans, etc. There are many methods and techniques in image processing, one of which is pattern recognition. Pattern recognition is a technique used to match a test image object with reference imagery. This technique is commonly used in the identification or recognition of object patterns such as fingerprints, faces, letters, numbers, and patterns of objects and so on [1].

Revised Manuscript Received on April 25, 2019.

Sufiatul Maryana, Department of Information System, Diploma Program Pakuan University, Indonesia.

Iyan Mulyana, Department of Computer Science, Faculty of Mathematics and Natural Science, Pakuan University, Indonesia.

Ema Kurnia, Department of Information System, Diploma Program Pakuan University, Indonesia.
Each letter written using handwriting must have different writing patterns, such as thickness and shape of the letter pattern. An object cannot just be analyzed without any approach technique. One of the techniques used in pattern recognition is the technique of Normalized Cross Correlation (NCC). NCC is a technique used to match two images to be compared by evaluating the level of difference or similarity of the two images. The result of comparison of image with NCC technique will get the value of correlation coefficient between -1 to 1 . An object can be said similar to referenceimage if the result of comparison has a value close to 1 .

Prior to pattern recognition, an object needs to go through the preprocessing stage. Convolution technique is one of preprocessing technique to perform image manipulation by using external mask to produce another image. Convolution techniques are widely used to refine, sharpen, and edge detection of image and more [2-3].

There have been several studies ever conducted on object recognition. Among them is the study of Kanata \& Bulkis [4]. The study makes fingerprints as objects and applies the NCC technique in the object recognition. The results stated that the maximum NCC value greater than 0.62 indicates the accuracy of the object's similarity.

According to the description above, this study will apply the techniques of normalized cross-correlation with the object used of hijaiyah letters. Object of hijaiyah letter will be done by preprocessing applying one method in convolution technique that is edge detection for subsequent to do pattern recognition object of hijaiyah letter and analyze the result [5].

\section{RESEARCH METHOD}

The research method used was a Normalized Cross Correlation (NCC). This method was used to get the value of correlation coefficient of the comparison between matching of two images compared that is the test image with the target image by evaluating the level of difference or similarity of the two images. Fig. 1 was a flowchart of the NCC Method. 


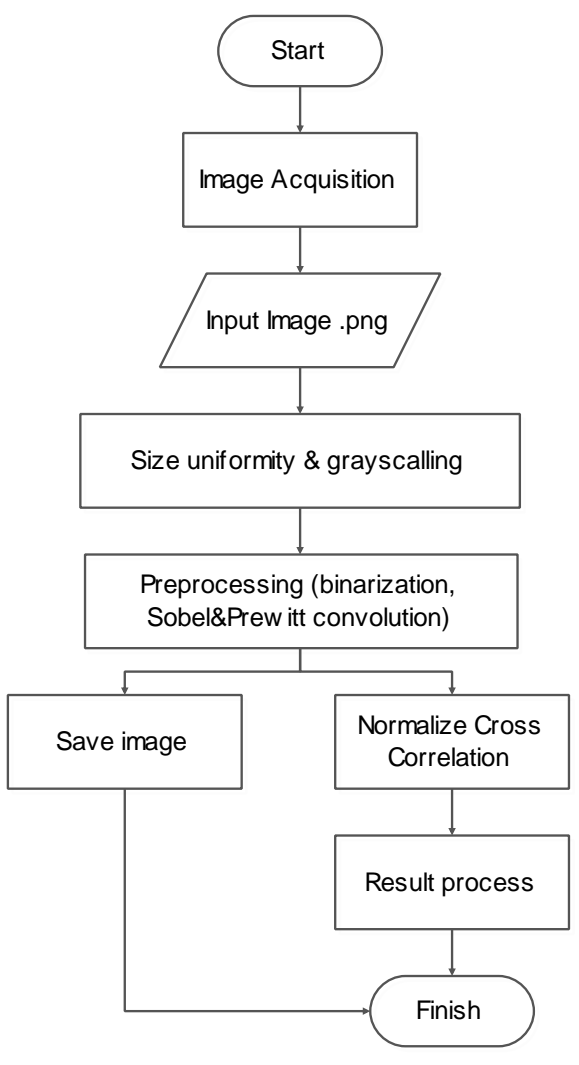

Fig. 1. Flow Chart of the NCC Method

\section{A. Image Acquisition}

Image acquisition was a step to record the image data for the next to be processed on computer. At this stage the data previously derived from the handwriting will be digitalized using the camera. Number of letters hijaiyah used 30 letters. Data sample used was 4 data sample handwriting with total amount is 120 letter data with extension of .png to be used as test image and target image as input.

\section{B. Size uniformity and gray scaling}

Size uniformity and gray scaling was the step taken to equalize the size of test image and target image before the binary and convolution process was done. The image size will be converted into $32 \times 32$ pixels and $64 \times 64$ pixels. After the image has the same size, the next step was to change the images into gray scale image.
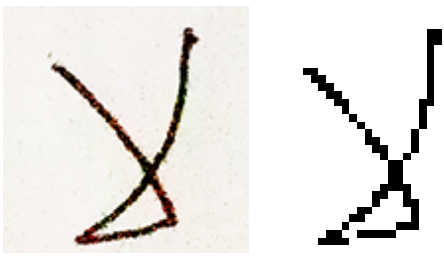

Fig. 2. Gray scale Image

\section{Preprocessing}

Preprocessing was the step before the image entering the main process stage that is NCC [6]. In this study the preprocessing was to convert the test image and the target image into binary and applied the convolution technique of Sobel and Prewitt edge detection. At this stage, the image that has been done preprocessing will be stored for further processing with NCC.
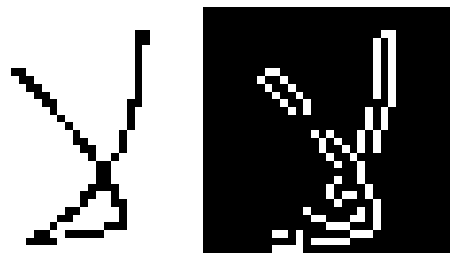

Fig. 3. Sobel Edge Detection Image

\section{Normalized Cross Correlation}

The normalized cross-correlation (NCC) was a step in getting the correlation coefficient value between the test image and the target image. This process was done after several previous stages of size uniformity, grayscaling, binary preprocessing and convolution technique of Sobel and Prewitt edge detection [7]. The equation in NCC as follow:

$$
r=\frac{\sum_{i=0}^{M-1} \sum_{j=0}^{N-1}\left(x_{i j}-\bar{x}\right)\left(y_{i j}-\bar{y}\right)}{\sqrt{\sum_{i=0}^{M-1} \sum_{j=0}^{M-1}\left(x_{i j}-\bar{x}\right)^{2} \cdot \sum_{i=0}^{M-1} \sum_{j=0}^{N-1}\left(y_{i j}-\bar{y}\right)^{2}}}
$$

where:

$r \quad$ : Coefficient correlation

$x$ : Target image

$\bar{x} \quad$ : Target image average value

$\bar{y} \quad$ : Average value of input image

$Y \quad$ : Input image

$M, N$ : Number of image pixel

The Flowchart of NCC as shown in Fig. 4.

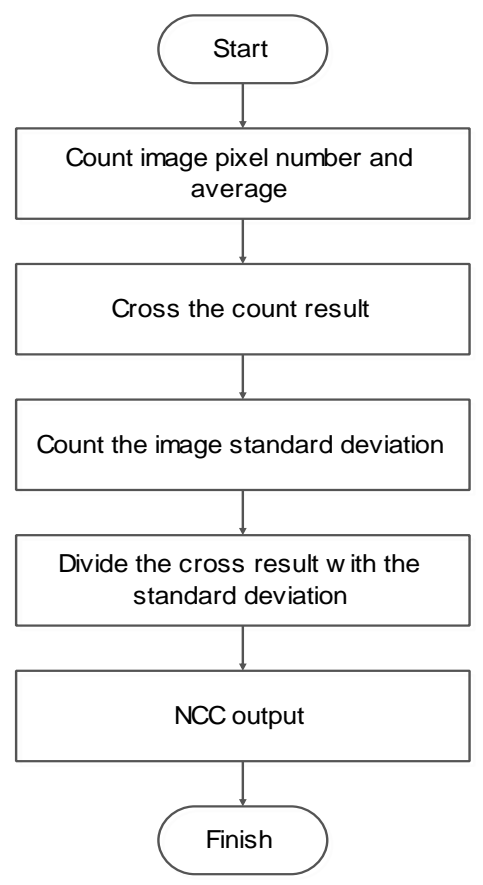

Fig. 4. Flowchart of NCC

Table 1 will be as a reference of correlation value to indicate the level of correlation or match between the target image and the test image.

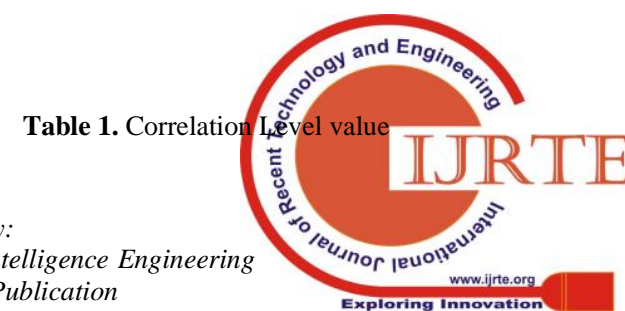




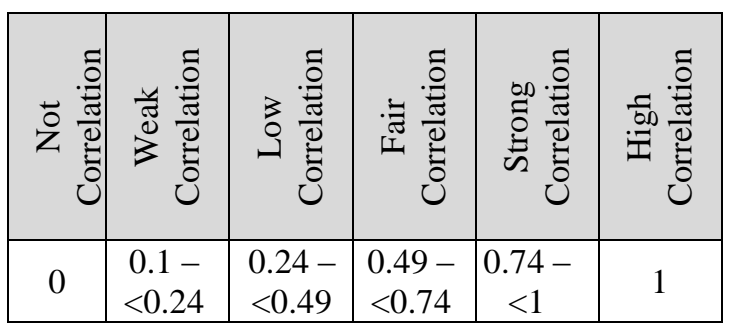

\section{E. Calculate the Number of Pixel and Average Image}

Calculated the number of pixels and average image was the initial stage in the normalized cross-correlation method. Before this stage was done, the test and target image are normalized by size uniformity of $64 \times 64$ pixels or $32 \times 32$ pixels and color uniformity into gray scale.

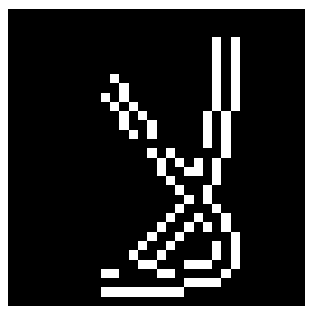

Fig. 5. Sobel Test Image (x)

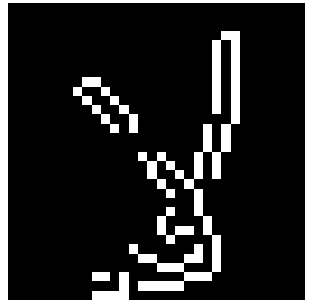

Fig. 6. Sobel Target Image (y)

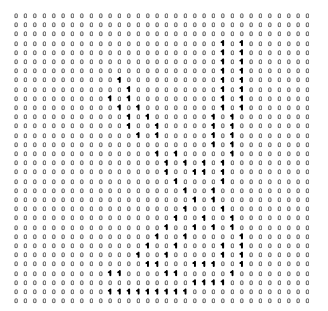

Fig. 7. Sobel Test Image Matrix (x)

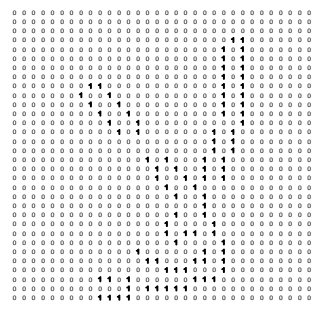

Fig. 8. Sobel Target Image Matrix (y)

Fig. 7 was a test image matrix with the number of pixels 97 to be compared with Fig. 8 which was the target image matrix with the number of pixels 92. The whole process was calculated using MATLAB. The number of pixels was derived from the total pixel value of 1 in the image. The result of comparison yields the mean target image value ( $\mathrm{rclb}$ ) and the average test image (rc2b) as follows:

$$
\bar{y}=\frac{92}{(32 \times 32)} \quad r c 1 b=0.0898 \quad \bar{x}=\frac{97}{(32 \times 32)} \quad r c 2 b=0.0947
$$

After the number of pixels and the average image was known, then subtracted each image pixel by the average image using equation 2 :

$$
\sum_{i=0}^{M-1} \sum_{j=0}^{N-1}\left(x_{i j}-\bar{x}\right)\left(y_{i j}-\bar{y}\right)
$$

The result of the process yields a new matrix as in Fig. 9 and Fig. 10:

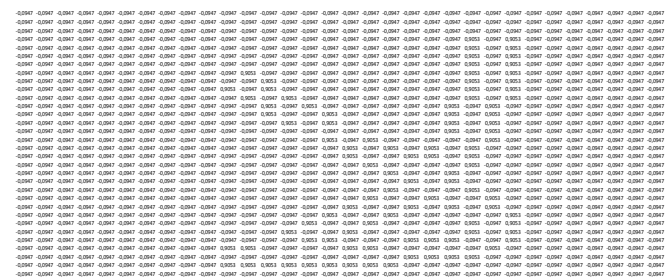

Fig. 9. New Test Image Matrixes

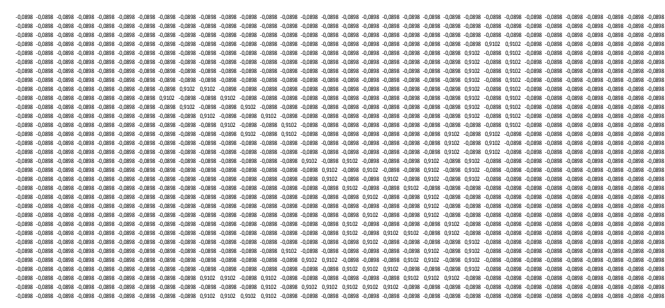

Fig. 10. New Target Image Matrixes

\section{F. Crossover Result Number and Average Image}

The stage of crossover results number and average images $(\mathrm{s} \lg 1 \mathrm{~b})$ was the stage done to cross the new test image and new target image matrix. The cross process was done by multiplying the new matrix from the test image and the target image with Equation 2 yields the value:

$$
s \lg 1 b=45.2852
$$

\section{G. Calculate Standard Deviation Image}

Calculated the standard deviation was the step taken to get the standard deviation value. This stage used the result of crossover number and average of images in the previous stage which are then included in the standard deviation formula by raised the new test image matrix and the new target image matrix to then at the square root. The process was done by using part of equation 3 :

$$
\sqrt{\sum_{i=0}^{M-1} \sum_{j=0}^{M-1}\left(x_{i j}-\bar{x}\right)^{2} \cdot \sum_{i=0}^{M-1} \sum_{j=0}^{N-1}\left(y_{i j}-\bar{y}\right)^{2}}
$$

The process results of equation 3 yields the standard deviation value $(\operatorname{stdv} 2)$ :

$$
\operatorname{std} v 2=85.7487
$$




\section{H. Dividing Crossover Result with the Standard Deviation result}

The dividing stage of the crossover result with the standard deviation result was the final stage of the normalized cross-correlation method. At this stage was to get the value of the correlation coefficient between the test image and the target image. At the process of test image and target image as in Fig. 4 and Fig. 5, the yield of correlation coefficient value (nc2) was:

$$
n c 2=0.5281
$$

The correlation coefficient value will be used as a benchmark whether the test image and the target image have a similarity. If it refers to Table 1 correlation level value, then the value of the test image correlation coefficient with the target image had fair correlation. Specifically, the test image pattern matches the target image.

\section{Process Result}

The process result will be displayed the output of the image that has been in preprocessing, which are the number of pixels test image and target image, the level of image matching, image coefficient correlation and the conclusion of the NCC process results was in the form of matches and unmatched image. As in the previous process results, the value of correlation coefficient was 0.5281 . This value was the final result of the process. Based on Table 1, the value of 0.5281 produces a "fair" match between the test image and the target image.

\section{RESULT AND DISCUSSION}

The testing was done by running the application by input and processed the image object. All testing was performed using 32x32 pixel and 64x64 pixel size image on binary and image of sobel and prewitt edge detection. The conducted test was:

\section{A. Testing with Similar Sample Data}

Testing with similar sample data was the test between test image and target image using the same sample data, letters and pattern. Based on the results of the overall Binary image, Sobel image and Prewitt image using similar sample data and same letter produced perfect accuracy and correlation. 100\% Accuracy and correlation value of 1 which means the overall test image object really match the target image. The test results can be concluded that the test image object with the same target image will produce a perfect match.

\section{B. Testing with Different Sample Data}

Testing with different sample data was a test between the test image and the target image using the sample data that has different writing patterns but with the same letter. Pixel average diagram of the test result with different sample data as shown in Fig. 11.

\section{Average Pixel of Test Image Point B (32×32 pixels)}

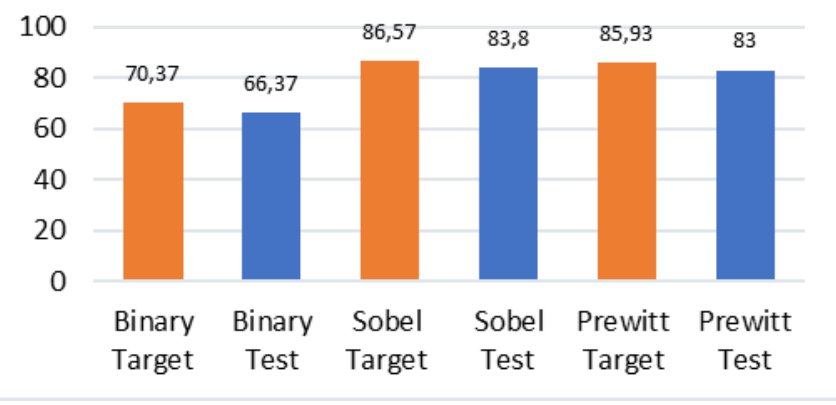

Fig. 11. Diagram of Average Pixel Image with Different Sample Data (32x32 pixels) (Gambar masih bhs Indonesia)

In the image with size $32 \times 32$ pixel produces accuracy on Binary image of $63.33 \%$ with 19 match letter, Sobel image of $46.67 \%$ with 14 match letter and Prewitt image of 50\% with 15 match letters.

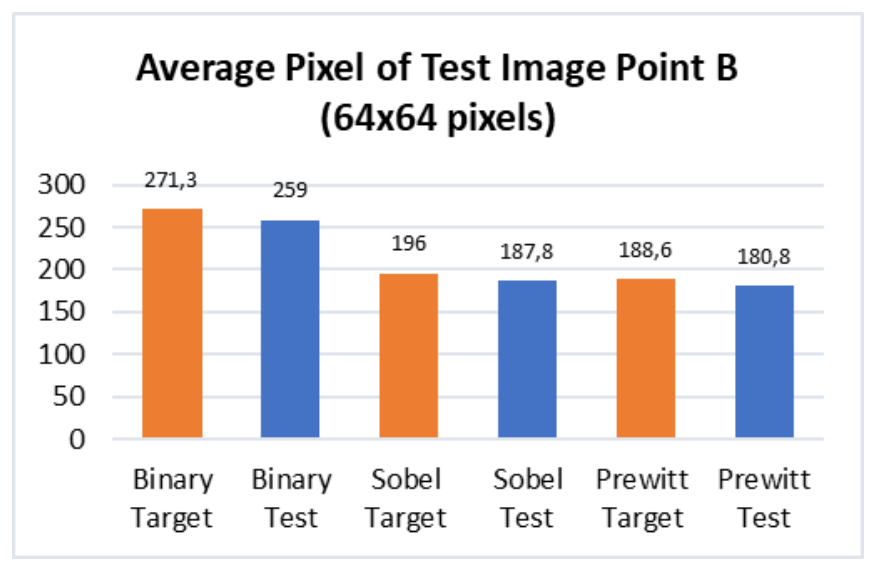

Fig. 12. Diagram of Average Pixel Image with Different Sample Data (64x64 pixels) (Gambar masih bhs Indonesia)

Unlike with the $64 \times 64$ pixel image, in the test of Binary image has $40 \%$ accuracy with 12 match letters, but in Sobel image and Prewitt image accuracy of $3.33 \%$ with 1 match letter. Fig. 11 and Fig. 12 represent the average image pixel calculation results obtained using Microsoft Excel 2013. In Fig. 11 the Binary image was lower than the Sobel and Prewitt images. In contrast to the Binary image in Fig. 12 that shows higher than the Sobel and Prewitt image. In general, the picture shows the average of total pixel of the target image was greater than the test image.

Based on the diagram, it shows that the target image that has a number of pixels larger or near the same, will have a good match. The matches were of course if between the test image and the target image has a similar pattern and small image will resulted in a high match letter. Unlike larger images, larger letter matching was found in Binary image compared with Sobel and Prewitt image. 


\section{Testing with sample data of different letter objects}

Testing with sample data of different letter objects was a test between test image with target image using different sample data or same sample data. For example, sample 2 and 3 data are Kho 2 with $\mathrm{Ja} 2, \mathrm{Na} 3$ with $\mathrm{Tsa} 3$ and so on. In this test used different letters but have similar patterns and fonts. Examples of letters such as Ro with Wa, Tho with Zo, and so forth. In all these tests was done to see how many objects matches between the test object and the target image both have little difference, or have many different patterns and objects of letters. The average pixel diagram of the test results as shown in Fig. 13.

\section{Average Pixel of Test Image Point C (32×32 pixels)}

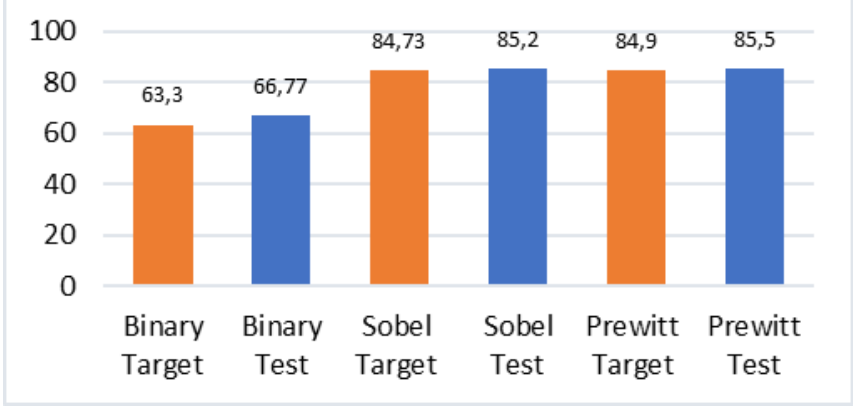

Fig. 13. Average Pixel Diagram of Test Image with Different Letters Sample Object Data (32 x 32 pixels)

The test of the $32 \times 32$ pixel image resulted in a lower accuracy than previous test with different sample data. The test resulted in an accuracy of a $40 \%$ Binary image with 12 matching letters, $20 \%$ Sobel image with 6 matching letters and $23.3 \%$ Prewitt image with 7 match letters.

\section{Average Pixel of Test Image Point C (64x64 pixels)}

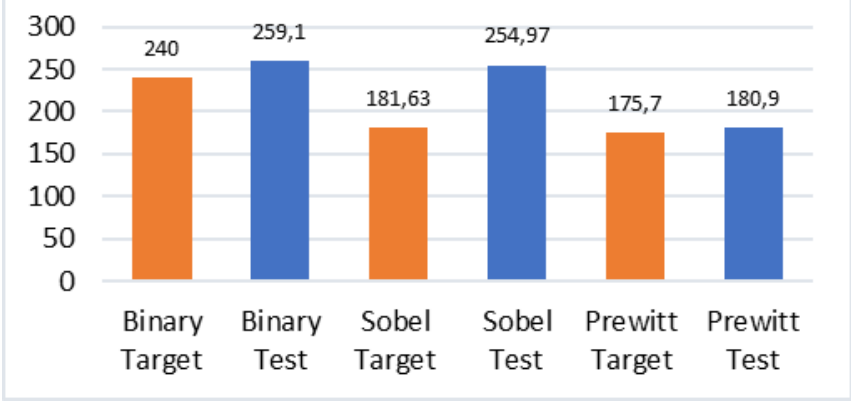

Fig. 14. Average Pixel Diagram of Test Image with Different Letters Sample Object data (64x64 pixels)

In contrast to a $64 \times 64$ pixel image, test resulted in lower accuracy. In the test, Binary image has an accuracy of $33.33 \%$ with 10 matching letters. But in the image with convolution of Sobel and Prewitt edge detection was not found any single letter that has a match. In Figs. 13 and 14 the total pixel average of all target images is lower than the test image. Likewise, with the result of the lowercase match number. Moreover, the test is done with different letter object but has similar pattern and shape of letters. The binary image still has more matching letters. But the matching results in imagery with Sobel and Prewitt edge detection convolution are very low, especially on $64 \times 64$ pixel sizes.

\section{Testing using Different Sample Data with Original Image Size}

Testing with sample data that has different writing patterns but with the same letter and using original image size of $125 \times 125$ pixels produced accuracy on binary image $43.33 \%$ with 12 match letters. But in the image with Sobel and Prewitt edge detection convolution not found any single letter that has a match. The average pixel diagram of the test results as shown in Fig. 15.

\section{Average Pixel of Test Image Point D (size of the original image)}

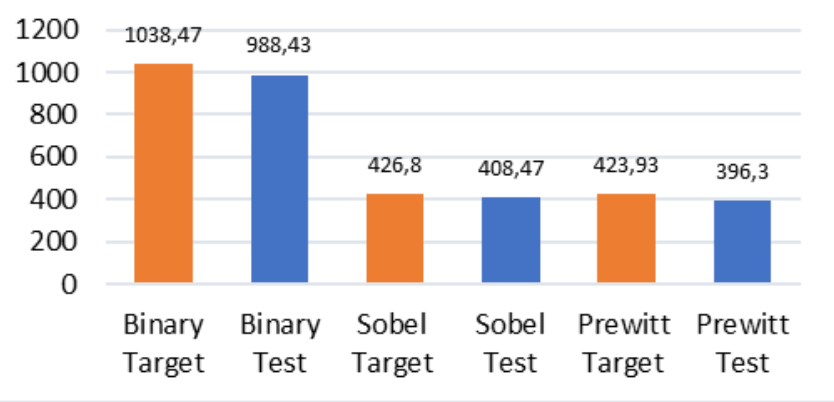

Fig. 15. Average pixel Diagram of tested image using sample data that different with original image size

Fig. 15 shows the average pixel of the target image higher than the test image. Binary imagery has an average number of pixels much higher than Sobel and Prewitt. When compared to Diagrams in Figs. 13 and 14 with Fig. 15, the average pixel number of target images of $125 \times 125$ pixels higher than the test image does not affect the number of matching letters.

Based on the trial results, experiments with different sample data have better results compared to the trials in the sample data differs from the original image size. In the overall result of the trial, matching letters was always higher in Binary image and in $32 \times 32$ pixel image compared to $64 \times 64$ pixels and $125 \times 125$ pixel images. While in the image with edge detection convolution of Sobel and Prewitt, the matching letters have a smaller number. This is because the pixel density level is 1 in the Binary image, denser than the image of Sobel and Prewitt. The target image and test image have a correlation value of 0.5228 . It assumed that the image position was at the point of intersection of 30.24. Then, a 1-pixel test image shifted to the right at the point of 30.25 , this shifted causes the image correlation value decreased to 0.2504 .

A sample of Sobel edge detection convolution image with a pixel value of 1 in the image was not too dense. Pixels value of 1 was just at the edge of the letters only. The target image and test image have a correlation value of 0.5281 . It assumed

that the image position was at the intersection point of 31.25. Then, a 1-pixel test image shifted to the left at the point of 31.24 , this shifted causes the inageanc5roestation value decrease drastically to 0.0966 . 
When compared to Binary image, the larger image and the image with edge detection convolution have more sensitive properties in matching letters. Because, every pixel of the image with edge detection played an important role in forming the letter object. Likewise, in the larger size image, pixel value of 1 will spread more broadly. A slight difference in the pixel position of value 1 between the test image and the target image, will affect a considerable correlation value. Therefore, the matching of Sobel and Prewitt image with large image yields a not-so-high value. If referring to Table 1, although the image of Sobel has a visual similarity, but if the method of normalized cross-correlation was applied then the object will be deemed to have very little pattern match because of the very small correlation coefficient. Unlike the 1 pixel shifted binary image, the matching pattern value was low. These causes, the image of Sobel and Prewitt convolution have high sensitivity in matching letters resulting in lower number of image matching. However, object matching with edge detection convolution techniques can be used for more precise object recognition rates due to their high sensitivity.

\section{CONCLUSION}

Hijaiyah letters recognition study with preprocessing convolution techniques was in desktop based. This application applied the normalized Cross Correlation method as well as the Sobel edge detection convolution techniques and Prewitt edge detection as its approach. Application trials were tested using different object sizes, trials using preprocessing of Sobel and Prewitt edge detection convolution techniques and trials with no preprocessing of convolution techniques using binary. The trial used 4 handwritten sample data. Each handwriting sample data is 30 letters, with a total of 120 data. Trials are performed with $32 \times 32$ pixels and 64x64 pixel imagery. Testing using sample data and letters between test images with the same target image will produce $100 \%$ accuracy. While the test using different sample data with the same letter produces $63.33 \%$ accuracy on Binary image, accuracy $46.67 \%$ in Sobel image and $50 \%$ accuracy on Prewitt image. While the test using the sample data object different letters, the accuracy of the image Binary $40 \%$, the image of $20 \%$ Sobel and $23.33 \%$ Prewitt image. In this study, the use of Prewitt edge detection convolution techniques has higher accuracy and matching results compared to Sobel imagery.

The test result showed that the matching of the hijaiyah letters tested depends on the size of the image and the similarity of the letter pattern. An image of 32x32 pixel size and similar in letter pattern will result in greater letter pattern match accuracy. In contrast to the $64 \times 64$ pixel image and to the original size of $125 \times 125$ pixels, it produced smaller letter pattern matching accuracy. The number of pixels in the target image also has an effect in matching objects. Target image with the number of pixels larger than the test image, of course, in letters that have similarity patterns will result in greater pattern matching. Binary image has higher letter matching because the pixel density of value 1 was more solid, unlike Sobel and Prewitt images that were only on the edge of letter objects.

\section{REFERENCES}

1. C. Saravanan and M. Surender. "Algorithm for face matching using normalized cross-correlation." International Journal of Engineering and Advanced Technology, vol. 2, no. 4, pp. 930-934, 2013.

2. W. Gazali, H. Soeparno and J. Ahliati. "Penerapan Metode Konvolusi Dalam Pengolahan Citra Digital." Jurnal Mat Stat Binus University, vol. 12, no. 2, pp. 103-113, 2012.

3. S. Kosasi, Rekonstruksi Degradasi dalam Pengolahan Citra Menggunakan Metode Konvolusi. Pontianak: STMIK Pontianak, 2015.

4. B. Kanata. "Pencocokan Citra Sidik Jari Menggunakan Korelasi Silang Ternormalisasi." Jurnal Rekayasa Elektrika, vol. 11, no. 4, pp. 144-148, 2015

5. I. A. Kanta. Pengenalan Pola Huruf Hijaiyah Tulisan Tangan Menggunakan Logika Fuzzy dengan Jaringan Syaraf Tiruan Backpropagation. Doctoral dissertation, Muhammadiyah University Surakarta, 2013.

6. V. Lusiana. "Deteksi Tepi pada Citra Digital menggunakan Metode Kirsch dan Robinson." Dinamik, vol. 18, no. 2, pp. 182-189, 2013.

7. S. A. A. Bowo. Analisis deteksi tepi untuk mengidentifikasi pola daun. Doctoral dissertation, Diponegoro University, 2011. 